\title{
Histological assessment of the Sydney classification of endoscopic gastritis
}

\author{
S I Khakoo, A J Lobo, N A Shepherd, S P Wilkinson
}

\begin{abstract}
To determine the significance of the endoscopic classification of gastritis proposed by a working party at the World Congress of Gastroenterology in Sydney 1990, 167 patients undergoing upper alimentary endoscopy were prospectively assessed by comprehensive endoscopic and histological methods. Ninety eight patients had endoscopic mucosal changes of gastritis according to the Sydney classification. Twenty six $(27 \%)$ of these had histologically normal biopsy specimens. This was not statistically significantly different to the $26(38 \%)$ of 69 with normal endoscopies whose biopsy specimens were histologically normal $\left(\chi^{2}=1 \cdot 857, \mathbf{p}>0 \cdot 1\right)$. Forty three $(62 \cdot 5 \%)$ patients with normal endoscopies had histological gastritis. No histological counterpart was found for the macroscopic appearances of the gastric mucosa said to show inflammation proposed by the Sydney classification of gastritis. These findings confirm the inappropriateness of an endoscopic diagnosis of gastritis and it is suggested such a term should be reserved for the histological findings.
\end{abstract}

(Gut 1994; 35: 1172-1175)

Up to $50 \%$ of the asymptomatic population may have histological evidence of gastritis ${ }^{12}$ and $45 \%$ of patients with dyspepsia have endoscopic appearances interpreted as gastritis. ${ }^{3}$ It has been suggested that the frequency of these mucosal abnormalities are under reported. ${ }^{45}$ Several endoscopic criteria said to point to gastritis have been suggested, with varying correlation to histological abnormalities. ${ }^{6-8}$ These include mucosal erythema, superficial breaks in the mucosa ('erosions'), and visible gastric vasculature. ${ }^{679-11}$ Histological lymphocytic gastritis has been suggested to correlate well with a distinct form of endoscopic abnormality, namely varioliform gastritis. ${ }^{12} 13$ Conversely it is well recognised that endoscopy may commonly be normal in the presence of histological gastritis. ${ }^{1415}$ The heterogeneity of published works on gastritis may be because of inconsistencies in the classification of the appearance of mucosal abnormalities, and to a paucity of biopsy material obtained at endoscopy. 4

The Sydney classification of endoscopic gastritis $^{4}$ aims to standardise reporting by classifying endoscopic gastritis into seven categories, based on 15 endoscopic mucosal features: oedema, punctate and confluent erythema, friability, punctate and confluent exudate, flat and raised erosions, rugal hyperplasia and atrophy, visibility of the vascular pattern, punctate and confluent intramural bleeding spots, and fine and coarse nodularity. It also re-categorises portal hypertensive gastropathy as congestive gastroenteropathy.

This study was devised to correlate the endoscopic features described by the Sydney system with the histological findings.

\section{Methods}

Two endoscopists participated in the study. One (SIK), a trainee in gastroenterology who had performed about 100 procedures himself carried out the assessment under the supervision of a consultant (SPW) with 20 years experience. The other (AJL) was a senior registrar in gastroenterology, who had performed more than 1500 procedures unsupervised. Any patient endoscoped by the above was eligible for inclusion, and no further selection criteria were invoked. All patients were questioned about the presence of dyspepsia, ${ }^{16}$ smoking and drinking habits, non-steroidal anti-inflammatory drugs (NSAIDs) and ulcer healing drug use, previous ulcer surgery, and previous endoscopy. An Olympus fibreoptic endoscope system was used, and standard 7 FG sized biopsy forceps were used to take biopsy specimens from the following sites as described by the Sydney system ${ }^{4}$ : two each from the anterior and posterior antrum, 2-5 $\mathrm{cm}$ from the pylorus; two each from the anterior and posterior body, $10 \mathrm{~cm}$ from the cardia; and two from any additional area of abnormality. A further antral biopsy specimen was taken for assessment for Helicobacter pylori by the urease test, Gram staining, and culture.

All endoscopies were reported according to the criteria of endoscopic gastritis described by the Sydney classification, which includes a subjective assessment of severity as mild, moderate or severe, ${ }^{4}$ and then classified into one of the following eight categories: erythematous/exudative gastritis, atrophic gastritis, raised erosive gastritis, flat erosive gastritis, haemorrhagic gastritis, rugal hyperplastic gastritis, enterogastric reflux gastritis, and congestive gastroenteropathy.

The eight biopsy specimens from each patient were prepared for histological assessment according to standard histopathological processes. Optical orientation was ensured at the embedding stage. Five $\mu \mathrm{g}$ paraffin sections were stained with haematoxylin and eosin. The histopathological features were reported according to the Sydney classification ${ }^{17}$ by one histopathologist (NAS) without knowledge of 
TABLE I Cases with two endoscopic diagnoses

\begin{tabular}{lll}
\hline Antrum diagnosis & Body diagnosis & No \\
\hline Raised erosive & Erythematous/exudative & 4 \\
Erythematous/exudative & Congestive gastritis & 4 \\
Atrophic & Erythematous/exudative & 3 \\
Erythematous/exudative & Atrophic & 2 \\
Atrophic & Haemorrhagic & 1 \\
Raised erosive & Atrophic & 1 \\
Raised erosive & Haemorrhagic & 1 \\
\hline
\end{tabular}

TABLE II Distribution of endoscopic findings

\begin{tabular}{llc}
\hline Diagnosis & $\begin{array}{l}\text { Antral } \\
\text { frequency }\end{array}$ & $\begin{array}{l}\text { Body } \\
\text { frequency }\end{array}$ \\
\hline Normal & 75 & 111 \\
Erythematous/exudative & 61 & 39 \\
Atrophic & 13 & 7 \\
Raised erosive & 12 & 1 \\
Enterogastric reflux & 3 & 2 \\
Congestive gastritis & 0 & 5 \\
Haemorrhagic & 0 & 2 \\
Flat erosive & 1 & 0 \\
\hline
\end{tabular}

the clinical or endoscopic findings. The endoscopic and histological findings were subsequently analysed and correlated using $\chi^{2}$ analysis, with Yates's correction where appropriate.

\section{PATIENTS}

A total of 167 patients were included in the study. These were referred from gastroenterology clinics, the wards, and as part of an open access service. Eighty eight patients were female and 79 male with a median age of 60 years (range 19-90). One hundred and thirty three patients had dyspeptic symptoms, 32 were taking ulcer healing drugs, 29 were taking NSAIDs including low dose $(75 \mathrm{mg})$ aspirin, and 49 patients smoked. Eleven patients had had previous surgery for peptic ulceration, and two of 11 had had gastric antrectomy.

Of the 167 patients endoscoped 24 had oesophagitis, 15 had active duodenal ulceration, and seven had active gastric ulceration. Eleven patients were thought by the endoscopist to have had excess bile in the stomach.

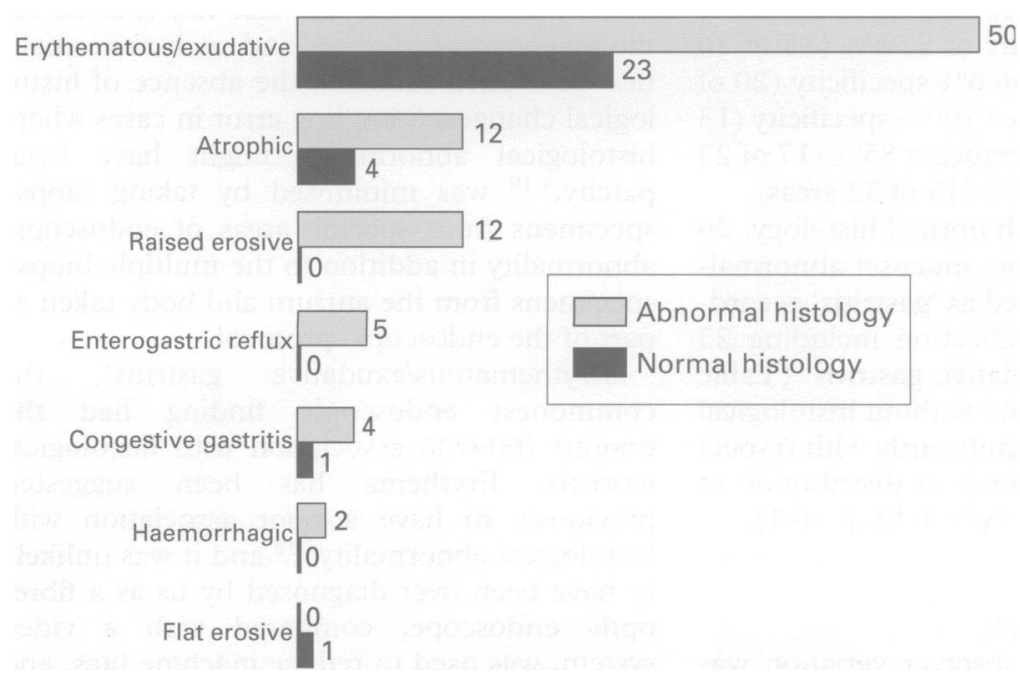

\section{Results}

ENDOSCOPY FINDINGS

In 98 of $167(58.6 \%)$ patients the following endoscopic mucosal changes considered by the Sydney classification to point to gastritis were found: erythematous/exudative gastritis 73 (44\%) patients, atrophic gastritis $16(10 \%)$, raised erosive gastritis $12(7 \%)$, enterogastric reflux gastritis five $(3 \%)$, congestive gastroenteropathy five (3\%), haemorrhagic gastritis two $(1.2 \%)$, and flat erosive gastritis one $(0 \cdot 6 \%)$. The commonest single mucosal abnormality was punctate erythema, which was found in $73(44 \%)$ patients. Sixteen patients had evidence of two different forms of 'endoscopic gastritis' affecting the antrum and body (Table I). Sixty nine (41\%) had no gastric mucosal abnormality.

The distribution of endoscopic mucosal change was predominantly antral in $41(42 \%)$ cases or pangastric $50(51 \%)$, being confined to the body in only seven (7\%) of cases (Table II).

The severity of the endoscopic mucosal changes was categorised as mild in $66(67 \%)$, moderate in $29(30 \%)$, and severe in only three cases $(3 \%)$.

There was no significant difference between the populations with and without 'endoscopic gastritis' with respect to age, sex, smoking, the presence of dyspepsia, previous surgery or NSAID use.

\section{HISTOPATHOLOGICAL FINDINGS}

One hundred and fifteen (69\%) cases had histological gastritis, classified as follows: Helicobacter pylori associated chronic active gastritis 71 (43\%), chronic active gastritis with no $H$ pylori detected nine $(5 \%)$, reactive gastritis $31(19 \%)$, mild to moderate atrophic gastritis $11(7 \%)$, severe, probably autoimmune associated, atrophic gastritis three $(2 \%)$, and chronic gastritis two $(1 \cdot 2 \%)$.

The distribution of histological gastritis was antral in $39(34 \%)$ cases and pangastric in $73(63 \%)$. Histological abnormalities were deemed to be mild in $34(30 \%)$ cases, moderate in $54(47 \%)$, and severe in $27(23 \%)$.

The $H$ pylori associated chronic active gastritis was antral in $13(18 \%)$, pangastric in $57(81 \%)$, and found in the body only in one $(1 \%)$ patient, who had had an antrectomy. Thirty $(42 \%)$ of these patients had an endoscopically normal mucosa.

\section{CORRELATION OF ENDOSCOPIC AND}

HISTOLOGICAL FINDINGS

Of the 98 cases of 'endoscopic gastritis' 26 (27\%) had normal histology, and 43 (62\%) of patients with normal endoscopies had abnormal histology. There was thus no significant correlation overall between the presence of endoscopic and histological gastritis $\left(\chi^{2}=1 \cdot 857\right.$ (Yates's correction), $\left.\mathrm{p}>0 \cdot 1\right)$.

Of the three most commonly found types of 'endoscopic gastritis', the association with histological gastritis was: erythematous/ 
TABLE III Overall correlation between macroscopic and microscopic findings (cases with two diagnoses are included twice)

\begin{tabular}{|c|c|c|c|c|c|c|c|c|}
\hline & & Normal & $\begin{array}{l}\text { HP positive } \\
\text { chronic active }\end{array}$ & Atrophic & Reactive & $\begin{array}{l}\text { HP negative } \\
\text { chronic, active }\end{array}$ & $\begin{array}{l}\text { Chronic } \\
\text { gastritis }\end{array}$ & Total \\
\hline \multirow[t]{2}{*}{ Normal } & antrum & 27 & 34 & 0 & 11 & 2 & 1 & 74 \\
\hline & body & 68 & 31 & 4 & 1 & 5 & 1 & 111 \\
\hline \multirow[t]{2}{*}{ Erythematous/exudative } & antrum & 20 & 25 & 1 & 11 & 2 & 2 & 60 \\
\hline & body & 19 & 15 & 3 & 3 & 1 & 0 & 41 \\
\hline \multirow[t]{2}{*}{ Atrophic } & antrum & 4 & 5 & 1 & 2 & 1 & 0 & 13 \\
\hline & body & 2 & 1 & 3 & 0 & 1 & 0 & 7 \\
\hline \multirow[t]{2}{*}{ Raised erosive } & antrum & 0 & 5 & 1 & 4 & 2 & 0 & 12 \\
\hline & body & 0 & 1 & 0 & 0 & 0 & 0 & 1 \\
\hline \multirow[t]{2}{*}{ Congestive } & antrum & 0 & 0 & 0 & 0 & 0 & 0 & 0 \\
\hline & body & 1 & 4 & 0 & 0 & 0 & 0 & 5 \\
\hline \multirow[t]{2}{*}{ Enterogastric reflux } & antrum & 0 & 2 & 0 & 1 & 0 & 0 & 3 \\
\hline & body & 0 & 0 & 0 & 0 & 1 & 0 & 2 \\
\hline \multirow[t]{2}{*}{ Haemorrhagic } & antrum & 0 & 0 & 0 & 0 & 0 & 0 & 0 \\
\hline & body & 1 & 1 & 0 & 0 & 0 & 0 & 2 \\
\hline \multirow[t]{2}{*}{ Flat erosive } & antrum & 1 & 0 & 0 & 0 & 0 & 0 & 1 \\
\hline & body & 0 & 0 & 0 & 0 & 0 & 0 & 0 \\
\hline
\end{tabular}

$H P=$ Helicobacter pylori.

TABLE IV Findings in the 17 patients endoscoped by both observers

\begin{tabular}{lll}
\hline Observer $A$ & Observer B & Frequency \\
\hline Normal & Normal & 4 \\
Erythematous/exudative & Normal & 2 \\
Erythematous/exudative & Erythematous/exudative & 6 \\
Erythematous/exudative & Flat erosive & 1 \\
Erythematous/exudative & Raised erosive + erythematous exudative & 1 \\
Raised erosive & Raised erosive & 1 \\
Atrophic & Normal & 1 \\
Congestive gastritis & Erythematous/exudative + congestive gastritis & 1 \\
\hline
\end{tabular}

exudative gastritis 50 of 73 patients $(68 \%)$, atrophic gastritis 12 of 16 patients (75\%), and raised erosive gastritis 12 of 12 patients $(100 \%)$, (Figure). The last category was significantly associated with histological gastritis $\left(x^{2}=4.39\right.$ (Yates's correction), $\left.\mathrm{p}<0.05\right)$, but not any specific type. Other endoscopic categories contained too few cases to draw meaningful conclusions (Table III).

No association was found between the severity of 'endoscopic gastritis' and the presence of histological abnormality $\left(\chi^{2}=2 \cdot 30\right.$, $\mathrm{p}>0 \cdot 1$ ).

Considering individual endoscopic findings in more detail, the commonest endoscopic abnormality was punctate erythema. This was found in 60 of 167 antrums and 34 of 116 bodies. Out of these 94 endoscopically distinct areas, 58 had associated histological gastritis. This gives a $61 \cdot 7 \%$ specificity of this abnormality for histological gastritis. Using the same criteria the presence of prominent lineae gastricae gave a specificity of $57 \cdot 6 \%$ (15 of 26 areas), visible vessels a $66 \cdot 6 \%$ specificity (20 of 30 areas), raised erosions a $100 \%$ specificity ( 13 of 13 areas), confluent erythema $85 \%$ (17 of 23 areas), and oedema $46.9 \%$ (15 of 32 areas).

Of the 52 patients with normal histology, 26 $(50 \%)$ had an endoscopic mucosal abnormality that would be classified as 'gastritis' according to the Sydney classification including 23 with 'erythematous/exudative gastritis' (Table III). The patients with and without histological gastritis did not differ significantly with respect to the endoscopic diagnosis or distribution of endoscopic abnormality $\left(x^{2}=3 \cdot 42, p>0 \cdot 1\right)$.

INTEROBSERVER VARIATION

An assessment of interobserver variation was performed on 17 patients. After intubation full inspection of the gastric mucosa was made by both operators. Table IV shows the results.

In 14 of 17 patients there was agreement on the presence or absence of 'endoscopic gastritis' as defined by the Sydney classification, and, of the 11 cases in which it was agreed to exist, there was complete agreement about the type of 'gastritis' in eight cases, and partial agreement in two cases.

In the 167 patients included there was no significant difference between the two operators in correlation between the endoscopic and histological findings in their respective endoscopies. Operator A had 60\% agreement between the presence of histological and endoscopic gastritis, and operator B 59\% agreement.

\section{Discussion}

No overall association was found between the endoscopic abnormalities described by the Sydney classification and histological gastritis. Both endoscopists participating in this study were in good agreement with their findings. This lack of association results from two main factors. Endoscopy is an insensitive method for diagnosing gastritis, and in our study despite the use of a comprehensive classification of gastritis we could not increase this sensitivity from the previously reported figures. ${ }^{518}$ The endoscopic abnormalities described in the Sydney classification ${ }^{4}$ are also not specific for the diagnosis of gastritis, and such abnormalities were often found in the absence of histological changes. Sampling error in cases where histological abnormality might have been patchy, ${ }^{419}$ was minimised by taking biopsy specimens from specific areas of endoscopic abnormality in addition to the multiple biopsy specimens from the antrum and body taken as part of the endoscopic protocol.

'Erythematous/exudative gastritis', the commonest endoscopic finding had the poorest $(60.6 \%)$ association with histological gastritis. Erythema has been suggested previously to have a poor association with histological abnormality ${ }^{4} 18$ and it was unlikely to have been over diagnosed by us as a fibreoptic endoscope, compared with a video system, was used to reduce machine bias, and the number of normal endoscopies associated 
with abnormal histology in our study was similar, or slightly greater, than has previously been reported. ${ }^{15} 2021$ Furthermore, erythema may not be reported consistently by independent observers. ${ }^{6}$ It may represent a transient vasodilatation of the gastric vasculature.

Apart from patients with end stage renal failure undergoing maintenance haemodialysis, ${ }^{22}$ endoscopic mucosal breaks ('erosions') have been reported to have a stronger association with histological gastritis than erythema. ${ }^{715}$ Our findings agree with this, as endoscopic 'raised erosive gastritis' had a $100 \%$ association with histological abnormality. It did not, however, have an association with any specific histological type of gastritis (Table III).

Nodularity of the mucosa has been proposed to show the presence of $H$ pylori in children ${ }^{23}$ and more recently in adults. ${ }^{24}$ We found a $76.9 \%$ association of nodules for $H$ pylori, not the $100 \%$ association reported previously in adults. These mucosal findings, and those categories of 'endoscopic gastritis' in which we had only small numbers constitute areas for further investigation.

No type of histological gastritis was specifically associated with a single category of endoscopic abnormality as described by the Sydney classification, although five of seven cases of visible vessels in the body were associated with histological atrophy.

Our study suggests that the endoscopic division of the Sydney classification has not helped to clarify the reporting of gastritis. Cases where nodularity, oedema or prominent lineae gastricae are found in isolation, are difficult to classify according to the Sydney system. The 15 mucosal findings it lists may be well employed as descriptive terms, but other than raised erosions, would not necessarily point to gastritis. Grouping endoscopic abnormalities into different categories of gastritis is misleading as they have no histopathological correlates. Misleading reports may lead to inappropriate treatment, especially with the increasing number of open access endoscopy units where reports must be interpreted by non-gastroenterologists. Endoscopy reports are more meaningful if the endoscopic mucosal findings are accurately described and biopsy specimens taken. We therefore suggest that the term 'gastritis' is reserved for histological abnormality.

1 Suirada M, Isokoski M, Vaius K, Kekki M. Prevalence of gastritis in a rural population. Scand f Gastroenterol 1978; 3: 211-23.

2 Johnsen R, Bernerson B, Straume B, Forde OH, Bostad L, Burhol P. Prevalence of endoscopic and histological findings in subjects with and without dyspepsia. $B M \mathcal{F}$ 1991; 302: 749-52.

3 Toukan AU, Kamal MF, Aur SS, Arnaout MA, AbuRomiyeh S. Gastroduodenal inflammation in patients with non ulcer dyspepsia. Dig Dis Sci 1985; 30: 313-20.

4 Tytgat GNJ. The Sydney system: endoscopic division endoscopic appearance in gastritis/duodenitis. $\mathcal{J}$ Gastroenterol Hepatol 1991; 6: 223-34.

5 Taor RE, Fox B, Ware J, Johnson AG. Gastritis gastroscopic and microscopic. Endoscopy 1975; 7: 209-15.

6 Sauerbruck F, Schreiker MA, Schusslen P, Permanetter W. Endoscopy in the diagnosis of gastritis, diagnostic value of endoscopic criteria in relation to histological diagnosis. Endoscopy 1984; 16: 101-4.

7 Fung M, Papadimitiou JM, Matz LR. Endoscopic, histological and ultrastructural correlations in chronic gastritis. Am $f$ Gastroenterol 1979; 71: 269-79.

8 Cronstedt JL, Simpson IW. Correlation between gastroscopic and direct vision biopsy. Gastrointest Endosc 1973; 19: 174-95.

9 Myren J, Serek-Hanssen A. The gastroscopic diagnosis of gastritis. Scand $\mathcal{F}$ Gastroenterol 1987; 22: 714-8.

10 Nesland AA, Berstad A, Serk Hanssen A. Histological findings in erosive prepyloric changes. Scand f Gastroenterol 1986; 21: 239-45.

11 Franzin G, Manfrui C, Musola R, Rodealla S, Fratton A. Chronic erosions of the stomach - a clinical, endoscopic and histological evaluation. Endoscopy 1984; 16: 1-5.

12 Haot J, Hanmich L, Wallez L, Mainguet P. Lymphocytic gastritis: a newly described entity: a retrospective endoscopic and histological study. Gut 1988; 29: 1258-64.

13 Lambert R, Andre C, Monlimi B, Bugnon B. Diffuse varioliform gastritis. Digestion 1978; 17: 159-67.

14 Vaira D, Holton J, Osborn J, D'Anna L, Romanos A, Falzon $M$, et al. Endoscopy in dyspeptic patients: is gastric mucosal biopsy useful? $A m \mathcal{F}$ Gastroenterol 1990;85 $701-4$.

15 Elta GH, Appleman HD, Behler EB. A study of the correlation between endoscopic and histological diagnosis in gastroduodenitis. Am $\mathcal{F}$ Gastroenterol 1987; 82: 353-8.

16 Colin-Jones DG. Management of dyspepsia: report of a working party. Lancet 1988; i: 576-9.

17 Price AB. The Sydney system: histological division. $f$ Gastroenterol Hepatol 1991; 6: 209-22.

18 Black DD, Haggitt RC, Whitington PF. Gastroduodenal endoscopic-histologic correlation in pediatric patients. Pediatr Gastroenterol Nutr 1988; 7: 353-8.

19 Gear MLW, Truelove SC, Whitehead R. Gastric ulcer and gastritis. Gut 1971; 12: 639-45.

20 Tytgat GNJ. Endoscopic gastritis and duodenitis. Endoscopy 1992; 24: 34-40.

21 Wee A, Kang JY, Ho MS, Choong HL, Wu AYT, Sutherland IH. Gastroduodenal mucosa in uraemia: endoscopic and histological correlation and prevalence of Helicobacter like organisms. Gut 1990; 31: 1093-6.

22 Kang JY, Wee A, Choong HL, Wu AYT. Erosive prepyloric changes in patients with end-stage renal failure undergoing maintenance dialysis treatment. Scand $\mathcal{F}$ Gastroenterol ing maintenance $25: 746-50$

23 Czinn SJ, Dahms BB, Jacobs GH, Kallen B, Rothstein FC. Campylobacter-like organisms in association with symptomatic gastritis in children. $f$ Pediatr 1986; 109: 80-3.

24 Grellier L, Tanner P, Grainger SL. Antral nodularity: macroscopic marker for Helicobacter pylori gastritis. Gut 1993; 34 (suppl 1): S35. 